\title{
COLOR IMAGE SEGMENTATION USING SOFT ROUGH FUZZY-C-MEANS CLUSTERING AND SMO SUPPORT VECTOR MACHINE
}

\author{
R.V.V.Krishna ${ }^{1}$ and S.Srinivas Kumar ${ }^{2}$ \\ ${ }^{1}$ Department of ECE, Sri Sai Aditya Institute of Science \& Technology, \\ Surampalem, Kakinada, India \\ ${ }^{2}$ Department of ECE, JNT University, Kakinada, Andhra Pradesh, India
}

\begin{abstract}
Color Image segmentation splits an image into modules, with high correlation among objects contained in the image. Many color image segmentation algorithms in the literature, segment an image on the basis of color, texture and as a combination of both color and texture. In this paper, a color image segmentation algorithm is proposed by extracting both texture and color features and applying them to the Sequential Minimal Optimization-Support Vector Machine (SMO-SVM) classifier for segmentation. Gabor filter decomposition is used for extracting the textural features and homogeneity model is used for obtaining the color features. The SMO-SVM is trained using the samples obtained from Soft Rough Fuzzy-C-Means (SRFCM) clustering. Fuzzy set based membership functions efficiently handle the problem of overlapping clusters. The lower and upper approximation concepts of rough sets effectively deal with uncertainty, vagueness, and incompleteness in data. Parameterization tools are not necessary in defining Soft set theory. The goodness aspects of soft sets, rough sets and fuzzy sets are integrated in the proposed algorithm to achieve improved segmentation performance. The proposed algorithm is comparable and achieved better performance compared with the state of the art algorithms found in the literature.
\end{abstract}

\section{KEYWORDS}

Classification, Clustering, Fuzzy Sets, Homogeneity, Rough Sets, Segmentation, Soft Sets, SMO-SVM classifier, Texture

\section{INTRODUCTION}

Color image segmentation [1] is a pre-processing step of prime importance, used in several computer vision and image processing related applications such as robotic vision, face recognition, Content Based Image Retrieval and medical imaging. It divides an image into distinct regions, such that pixels have peak value of similarity index in each region and peak value of contrast index between regions. Image properties such as gray-level, intensity, and texture are used to recognize similar regions and similarity of such properties is used to construct groups of regions. [3]. Image segmentation algorithms can be categorized into four major groups, thresholding, clustering, edge based and region based segmentation.

Clustering techniques are explored in recent times for color image segmentation. Xiang Yang Wang et al., in their work [19] applied the pixel wise homogeneity and texture features to SVM

DOI : $10.5121 /$ sipij.2015.6504 
Signal \& Image Processing : An International Journal (SIPIJ) Vol.6, No.5, October 2015

by training SVM, using the features obtained by preliminary clustering with Fuzzy C Means (FCM) algorithm [21]. P. Lingras [8] et al., proposed rough $\mathrm{k}$ means algorithm for use in clustering of internet users, which was later applied for image segmentation applications.

Hong Ying Yang et al, [20] extracted pixel level colour features by considering local HVS model. The extracted features were then applied to Least Squares Support Vector Machine (LS-SVM) using Arimito entropy thresholding. Subrajeeth Mohapatra et al., presented their work on unsupervised Leukocyte segmentation using Rough Fuzzy Clustering. M. M. Mushrif and Ajay. K. Ray described an Intuitionist Fuzzy Sets dependent multi-thresholding algorithm for colour segmentation. [11,12] Vitronino and Fernando proposed colour image segmentation using "Genetic algorithm and Fuzzy C means". Rocio A. Lizarraga-Morales et al.,[14] proposed a segmentation method in which the color and texture features are combined using a rough setbased technique. Freixenet et al., [5] proposed to integrate the information pertaining to region and boundary for colour texture based segmentation. They experimented and obtained the initial seeds from the regions, by considering perceptual colour and texture edges. Deng et al., [3] proposed the well known J-SEGmentation (JSEG) algorithm, which combined quantization process and clustering techniques for extraction of colour-texture cues in images. Mean Shift clustering in sync with edge information was employed by christoudias et al.,[2] in their work on edge detection and image segmentation (EDISION) system. Colour and texture [17] cues play a predominant rule in segmenting the image. The segmentation algorithms based on clustering are unsupervised and so avoid human intervention.

In this paper, Color image segmentation technique using Soft Rough Fuzzy C Means using SMO$S V M$ is presented. Initially the color and texture cues of the colour image, at pixel level are obtained through homogeneity and Gabor filter. These features are then applied to Soft Rough Fuzzy C means (SRFCM) clustering algorithm. Later the SMO SVM classifier is trained by using samples obtained from SRFCM clustering. The color image is segmented with trained SVM model (classifier) in the final step. The pixel level information of color image coupled with classification capability of SMO-SVM classifier is the major strength of this technique. Simulated results show that the proposed method achieves better segmentation results. Performance measures compared with state of the art algorithms has been discussed in this paper.

The organization of the paper is as follows. The preliminaries of SRFCM clustering are discussed in Section 2. The basic concepts of SVM and SMO-SVM are discussed in section 3. In section 4 the proposed Color image segmentation using SRFCM clustering and SMO-SVM is discussed, followed by justification for using this algorithm. In Section 5 the performance measures used in evaluating the segmentation algorithm are presented. Section 6 shows the pictorial and objective evaluation results of the proposed algorithm. The concluding remarks are given in section 7 .

\section{SOFT ROUGH FUZZY C-MEANS ALGORITHM (SRFCM)}

SRFCM has its roots in the k-means algorithm proposed by J Mc Queen. This algorithm assigns objects to the nearest cluster by distance. Later Fuzzy C-Means (FCM) Algorithm was proposed by Bezdek. In FCM, objects are not confined to belong to a single cluster. Each object belongs to all clusters with certain degree of belongingness. Rough k-means (RKM) was proposed by Lingras and West [8] by borrowing some of the concepts of rough set theory [13], but not all core concepts. Rough Fuzzy c-means algorithm was proposed by S. Mitra et al., [10] and they applied 
Signal \& Image Processing : An International Journal (SIPIJ) Vol.6, No.5, October 2015

the algorithm to medical image segmentation problem In this paper SRFCM is proposed by applying similarity concepts of soft sets to Rough Fuzzy Frame work.

In a segmentation problem, the task is to know whether two objects are similar, and a measure of their similarity is needed for clustering different objects in an image.Many authors were intrigued, and mined the issue of similarity measurement between sets. P. Majumdar and S.K. Samanta [9] presented the theory of similarity measurement of soft sets as follows.

Let $U=\left\{o_{1}, o_{2}, \ldots . o_{m}\right\}$ be the set of objects and

$P=\left\{p_{1}, p_{2}, \ldots p_{n}\right\}$ be the set of parameters.

$\hat{Q}=\left\{F\left(o_{i}\right), i=1,2, \ldots m\right\}$ and

$\hat{R}=\left\{G\left(p_{i}\right), i=1,2, \ldots . n\right\}$ be two groups of fuzzy soft sets.

The similarity between $\hat{Q}$ and $\hat{R}$ is denoted by $\mathrm{S}(\hat{Q}, \hat{R})$ and is given by the following expression. $S(\hat{Q}, \hat{R})=\max S_{i}(\hat{Q}, \hat{R}) \quad$ where

$$
S_{i}(\hat{Q}, \hat{R})=1-\frac{\sum_{j=1}^{n}\left|\hat{Q}_{i j}-\hat{R}_{i j}\right|}{\sum_{j=1}^{n}\left|\hat{Q}_{i j}+\hat{R}_{i j}\right|}
$$

The fuzzy soft set based similarity technique is applied to compute the similarity of objects in images. Only maximum similarity pixels are placed in same cluster and low similarity pixels are placed in different clusters. The soft set similarity proposed by P. Majumdar and S. K. Samanta is adapted to the Rough Fuzzy C-Means algorithm by considering that $\hat{Q}$ is the soft set representing the samples and $\hat{R}$ is the soft set representing cluster centroids.

The fundamental steps of SRFCM are as follows.

1. Assume $m$ random initial cluster prototypes denoted by $c_{i}$.

2. Find membership $u_{i k}$ between $m$ clusters and $k$ data points.

3. Allocate each data point $o_{k}$ to the lower approximation $\left(\underline{A} U_{i}\right)$ or upper approximation ( $\bar{A} U_{i}$ and $\bar{A} U_{j}$ ) by computing $u_{i k}-u_{j k}$, where $u_{i k}$ be highest and $u_{j k}$ be second highest membership of a data point $o_{k}$ among all the clusters. A data point can belong to at most one lower approximation, and may belong to two or more upper approximations.

4. If the difference between the highest and next highest membership of a data point in all the clusters i.e $\left(u_{i k}-u_{j k}\right)$ is below some pre-defined threshold value, then $o_{k} \in \bar{A} U_{i}$ and $0_{\mathrm{k}} \in \overline{\mathrm{A}} \mathrm{U}_{\mathrm{j}}$. It also implies that $\mathrm{o}_{\mathrm{k}}$ cannot be a member of any lower approximation. On the other hand if $\left(u_{i k}-u_{j k}\right)$ is above the threshold value then $o_{k} \in \underline{A} U_{i}$ which implies that membership value $u_{i k}$ is highest among all the $m$ clusters.

5. Compute similarity of sample points soft set to the cluster center soft set by using the given formula. 
Signal \& Image Processing : An International Journal (SIPIJ) Vol.6, No.5, October 2015

$$
S_{i}(\hat{O}, \hat{V})=1-\frac{\sum_{j=1}^{n}\left|\hat{O}_{i j}-\hat{V}_{i j}\right|}{\sum_{j=1}^{n}\left|\hat{O}_{i j}+\hat{V}_{i j}\right|}
$$

Calculate the maximum similarity and assign a pixel to a cluster to which it has maximum similarity after fuzzification.

6. Compute updated cluster prototype for each cluster $U_{i}$, as in (3).

$$
\begin{aligned}
& v_{i}= \begin{cases}M_{1}, & \text { if } \underline{A} U_{i} \neq \Phi \wedge \bar{A} U_{i}-\underline{A} U_{i} \neq \Phi, \\
M_{2}, & \text { if } \underline{A} U_{i}=\Phi \wedge \bar{A} U_{i}-\underline{A} U_{i} \neq \Phi, \\
M_{3} & \text { otherwise }\end{cases} \\
& M_{1}=w_{l o w} \times \frac{\sum_{O_{k} \in \underline{\underline{A}} U_{i}^{m}} u_{i k}^{m}}{\sum_{o_{k} \in \underline{B} U_{i}}^{m} u_{i k}}+w_{u p} \times \frac{\sum_{o_{k} \in \bar{A} U_{i}-\underline{\underline{A}} U_{i}} u_{i k}^{m} o k}{\sum_{o_{k} \in \bar{A} U_{i}-\underline{\underline{A}} U_{i}} u_{i k}^{m}} \\
& M_{2}=\frac{\sum_{o_{k} \in \bar{A} U_{i}-\underline{A} U_{i}} u_{i k}^{m} o k}{\sum_{o_{k} \in \bar{A} U_{i}-\underline{A} U_{i}} u_{i k}^{m}} \\
& M_{3}=\frac{\sum_{O_{k} \in \underline{A} U_{i}} u_{i k}^{m} o_{k}}{\sum_{O_{k} \in \underline{A} U_{i}} u_{i k}^{m}}
\end{aligned}
$$

7. Iterate and run steps $2-6$ until there are no further changes in cluster centroids. The weights $\left(w_{\text {up }}, w_{\text {low }}\right)$ are chosen to be values between 0 and 1 .Further $w_{\text {up }}=1-w_{\text {low }}, 0.5<$ $w_{\text {low }}<1,0<T<0.5$.

\section{SUPPORT VECTOR MACHINE}

\subsection{Linear and Non-Linear SVM}

Support vector machine (SVM) [18] in general is used to solve classification problems encountered in pattern recognition. Two class SVM is used to divide data into two sets of classes, by estimating the location of a hyper plane that optimizes (increases) the minimum distance between any two groups as shown in Figure.1.

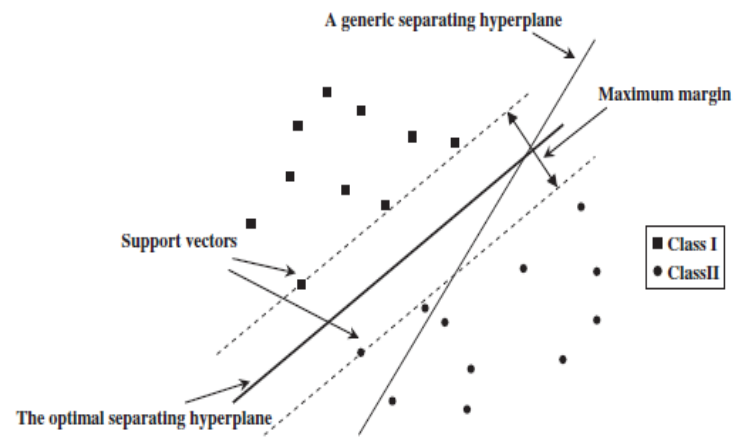

Figure1.SVM classification (linear) 
Signal \& Image Processing : An International Journal (SIPIJ) Vol.6, No.5, October 2015

Different hyper planes separate the data, but the hyper plane that optimizes the distance $2 / \mathrm{w}$ between the classes has to be found. SVM is not limited to just linear classification problems, but can also be applied to non linear classification problems. SVM tools are used in image processing to classify a feature set, derived from the image using annotated feature vectors. SVM classifiers come under the category of supervised methods, as they require training data which are manually annotated. The training data is used as reference for automatic classification of unclassified data. Let the training data be $\left(x_{i}, y_{i}\right)$ and the corresponding output be $y_{i} \in(-1,+1)$. SVM is modelled as

$y=w^{T} x+b$

where $\mathrm{b}$ is bias and $\mathrm{w}$ is weighted vector of the same dimensions as feature space.

SVM is formulated by assuming that given data can be linearly separated as given below.

$$
\begin{aligned}
& w^{T} x_{i}+b \geq+1\left(y_{i}=+1\right) \\
& w^{T} x_{i}+b \geq-1\left(y_{i}=-1\right)
\end{aligned}
$$

The margin $m$ is thus

$m=\frac{1}{\|\bar{w}\|^{2}}$

Maximum margin implies minimum w, and the problem is solved as follows

$\min _{w, b} \frac{1}{2}\|w\|$ with the constraint

$y_{i}(\bar{w} \cdot \bar{x}-b) \geq 1 \quad \forall i$

where $x_{i}$ is the $i^{\text {th }}$ training data point and $y_{i}$ is the expected response of the SVM for $i^{\text {th }}$ training data point. The value of $y_{i}$ is +1 for the excitations from group 1 and -1 for excitations from group 2.

The optimization problem can be solved by using Lagrangian. The cost function is dependent on the Lagrangian multipliers $\alpha_{i}$ as shown below.

$$
\min _{\alpha} \psi(\bar{\alpha})=\min _{\alpha} \frac{1}{2} \sum_{i=1}^{N} \sum_{j=1}^{N} x_{i} y_{j}\left(\bar{x}_{i} \cdot \bar{x}_{j}\right) \alpha_{i} \alpha_{j}-\sum_{i=1}^{N} \alpha_{i}
$$

$\mathrm{N}$ is no. of training examples subject to the constraints $\alpha_{\mathrm{i}} \geq 0$ and

$\sum_{i=1}^{N} y_{i} \alpha_{i}=0$

SVM's can be extended to nonlinear classifiers as well. The output of a non-linear SVM is defined in terms of Lagrangians as 
$u=\sum_{j=1}^{N} y_{j} a_{j} k\left(\overline{x_{j}}, \bar{x}\right)-b$

Where $\mathrm{k}$ is kernel that signifies the proximity of input $\bar{x}$ and annotated input $\bar{x}_{j}$.

The Lagrange multipliers ais are estimated by finding a solution to the quadratic equation. The non-linear variables change the quadratic form, still the cost function $\Psi$ maintains quadratic form as a function of $\alpha_{i}$ :

$$
\begin{aligned}
& \min _{\alpha} \psi(\alpha)=\min _{\alpha} \frac{1}{2} \sum_{i=1}^{N} \sum_{j=1}^{N} y_{i} y_{j}\left(\bar{x}_{i} \cdot \bar{x}_{j}\right) \alpha_{i} \alpha_{j}-\sum_{i=1}^{N} \alpha_{i} \\
& 0 \leq \alpha_{i} \leq c \quad \forall i \text { (box constraint) }
\end{aligned}
$$

The Quadratic programming problem in the above equation is solved by SMO Algorithm.

\subsection{Sequential Minimal Optimization (SMO) SVM}

SVM training intends to find a solution to a larger quadratic programming (QP) task. SMO splits a large QP into an array of smaller QP problems. The resulting small QP problems can be solved in lesser time compared with numerical quadratic programming. The memory used by SMO [6] is less in comparison with SVM so, it can efficiently deal with very large training sets. SMO achieves higher speed for linear SVM'S.

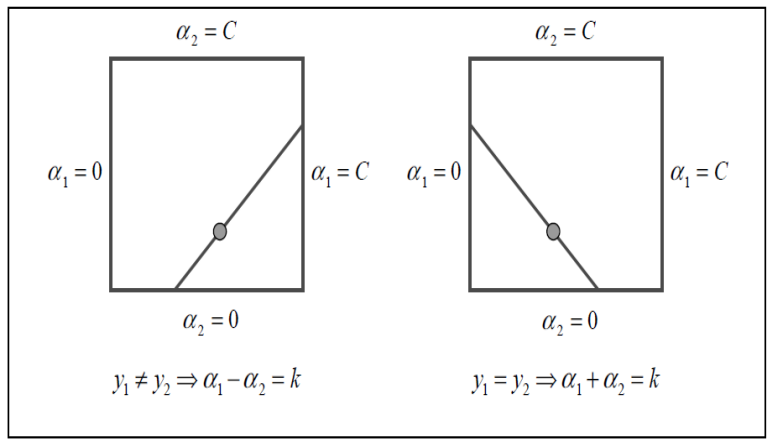

Fig.2.Two Lagrange multipliers

In SMO the kernel function $\mathrm{k}$ is bound to follow Mercer's conditions to maintain QP problem eternal positive.

The QP problem ends in a solution for every value of $i$, when

$$
\begin{array}{ll}
\alpha_{i}=0 & \Leftrightarrow \quad y_{i} u_{i} \geq 1 \\
0<\alpha_{i}<c & \Leftrightarrow \quad y_{i} u_{i}=1 \\
\alpha_{i}=c & \Leftrightarrow \quad y_{i} u_{i} \leq 1
\end{array}
$$

$\mathrm{u}_{\mathrm{i}}$ is the response for $\mathrm{i}^{\text {th }}$ training vector. 
Signal \& Image Processing : An International Journal (SIPIJ) Vol.6, No.5, October 2015

The Lagrange multipliers are found by solving for constrained minimum. The two Lagrangians, can be shown as a two dimensional function. The bound constraint (6) fits the Lagrange multipliers within a box, and the equality constraint confines the Lagrangian multiplier to lie on a diagonal line. Thus, the conditional minimum of the cost function is also bound to lie on a diagonal line.

If the targets $y_{1}$ and $y_{2}$ do not match, then the bounds defining $\alpha_{2}$ are.

$L=\max \left(0, \alpha_{2}-\alpha_{1}\right), H=\min \left(c, c+\alpha_{2}-\alpha_{1}\right)$

In the alternate case bounds defining $\alpha_{2}$ are

$$
\begin{aligned}
& L=\max \left(0, \alpha_{2}+\alpha_{1}-c\right), H=\min \left(c, \alpha_{2}+\alpha_{1}\right) \\
& \alpha_{2}^{\text {new }}=\alpha_{2}+\frac{y_{2}(E 1-E 2)}{\eta} \\
& \eta=k\left(\overline{x_{1}}, \overline{x_{1}}\right)+k\left(\overline{x_{2}}, \overline{x_{2}}\right)-2 k\left(\overline{x_{1}}, \overline{x_{2}}\right) \quad \eta>0
\end{aligned}
$$

where $e_{i}=u_{i-} v_{i}$ is the error in $\mathrm{i}^{\text {th }}$ training sample.

The constrained minimum is computed by fixing the unconstrained minimum to the ends of the line segment in next stage.

$$
\begin{array}{rlrrr}
\alpha_{2}^{\text {new } \text {,clipped }} & =H & \text { if } & \alpha_{2}^{\text {new }} \geq H \\
= & \alpha_{2}^{\text {new }} \text { if } & L<\alpha_{2}^{\text {new }}<H \\
= & L \text { if } & \alpha_{2}^{\text {new }} \leq L
\end{array}
$$

If $s=y_{1} y_{2}$. The value of $\alpha_{1}$ is

$$
\alpha_{1}^{\text {new }}=\alpha_{1}+s\left(\alpha_{2}-\alpha_{2}^{\text {new }} \text {, clipped }\right)
$$

\section{IMAGE SEGMENTATION USING SRFCM AND SMO SVM (PROPOSED METHOD)}

\subsection{Algorithm Steps}

1. Color, texture and spatial feature cues are extracted from the image. Homogeneity model is used to extract color features and Gabor filter decomposition for texture features. Additionally the spatial information is embedded in the feature vector to nullify the effect of noise and outliers.

2. SRFCM based clustering is applied on the feature space for selecting the training samples which are to be applied to the SMO-SVM classifier in the next stage of segmentation. 
Signal \& Image Processing : An International Journal (SIPIJ) Vol.6, No.5, October 2015

3. SMO-SVM training

The SMO-SVM classifier is trained using samples obtained from preceding step. For image pixels in $\mathrm{j}^{\text {th }}$ cluster some pixels are chosen as training samples remaining are used as test samples.

4. SMO SVM pixel classification

Apply the test set to SMO SVM for classifying new data. Combine test set and training set to obtain segmentation result.

\subsection{Colour Feature Calculation}

All the pixels in the image are marked as homogenous region pertaining to an object. The image segmentation task is now a classification problem and the process of segmentation is aimed at assigning a label to each individual pixel or an entire region based on homogeneity. Color features are extracted from the Lab color model, because color difference can be measured conveniently in $L A B$ color space.

Let $C_{i j}=\left(C_{i j}{ }^{\mathrm{L}}, C_{i j}{ }^{a}, C_{i j}{ }^{b}\right)$ be the representation of color components in Lab colour model, corresponding to a pixel at the point $(\mathrm{i}, \mathrm{j})$ in an image. The color feature $\mathrm{CF}_{\mathrm{ij}}{ }^{\mathrm{k}}, \mathrm{k}=\mathrm{L}, \mathrm{a}, \mathrm{b}$ is derived from the color component $\mathrm{C}_{\mathrm{ij}}{ }^{\mathrm{k}}, \mathrm{k}=\mathrm{L}, \mathrm{a}, \mathrm{b}$ as follows.

1. Prepare a window of size $3^{\times} 3$ for construction of pixel-level color feature.

2. Calculate pixel wise color feature $\mathrm{CF}_{\mathrm{ij}}{ }^{\mathrm{k}}$ related to the color component $\mathrm{C}_{\mathrm{ij}}{ }^{\mathrm{k}}$, using pixel homogeneity, extracted from image, so that it reflects the uniformity of an image object. Pixel variance in terms of standard deviation and discontinuity in terms of edge detection, of color component $\mathrm{C}_{\mathrm{ij}}{ }^{\mathrm{k}}$ are calculated. The product of normalized standard deviation and normalized edge discontinuity information is deducted from unity to obtain pixel homogeneity of the objects in the image.

Standard deviation and mean are defined as shown below. They are defined for each color feature $\mathrm{C}_{\mathrm{ij}}^{\mathrm{k}}(\mathrm{k}=\mathrm{L}, \mathrm{a}, \mathrm{b})$ at location $(\mathrm{i}, \mathrm{j})$.

$v_{i j}^{k}=\sqrt{\frac{1}{d^{2}} \sum_{m=i-\left(\frac{d-1}{2}\right)}^{i+\left(\frac{d-1}{\sum^{2}}\right)} \sum_{n=j-\left(\frac{d-1}{2}\right)}^{j+\left(\frac{d-1}{2}\right)}\left(c_{m n}^{k}-\mu_{i j}^{k}\right)^{2}}$

and

$\mu_{i j}^{k}=\frac{1}{d^{2}} \underset{m=i-\left(\frac{d-1}{2}\right)}{i+\left(\frac{d-1}{2}\right)} \sum_{n=j-\left(\frac{d-1}{2}\right)}^{j+} c^{c_{m n}^{k}}$

where $\mu_{i j}^{k}$ is mean of color component $c_{i j}^{k}(k=L, a, b)$

The edge variations are calculated in terms of the absolute value of first order derivative.

Let $\mathrm{e}_{\mathrm{ij}}{ }^{\mathrm{k}}, \mathrm{k}=\mathrm{L}, \mathrm{a}, \mathrm{b}$ represent the gradient operator at a point $(\mathrm{i}, \mathrm{j})$ in the image. Gradient operator indicates the rate of change at any point in the image.

$e_{i j}^{k}=\sqrt{\left(G_{x}^{k}\right)^{2}+\left(G_{y}^{k}\right)^{2}}$ 
$\left(G_{x}^{k}\right)$ and $\left(G_{y}^{k}\right)$ are composed of gradient components in $\mathrm{x}$ and $\mathrm{y}$ dimensions

$$
\begin{aligned}
& V_{i j}{ }^{k}=\frac{v_{i j}^{k}}{v_{\max }^{k}}, E_{i j}^{k}=\frac{e_{i j}^{k}}{e_{\max }^{k}} \\
& \text { where } v_{\max }^{k}=\max \left\{v_{i j}^{k}\right\}, e_{\max }^{k}=\max \left\{e_{i j}^{k}\right\},(0 \leq i \leq M-1,0 \leq j \leq N-1), k=L, a, b
\end{aligned}
$$

The colour feature is expressed as

$$
C F_{i j}^{k}=H_{i j}^{k}=1-E_{i j}^{k} \times V_{i j}^{k},(0 \leq i \leq M-1,0 \leq j \leq N-1), k=L, a, b
$$

\subsection{Texture Feature Calculation}

Texture is an important cue used in image segmentation. The combination of color and texture is used to achieve better segmentation results than possible with color just alone[16]. Gabor filter, used to extract the texture feature, [7] is a steerable filter, which operates at different scales and orientations to obtain the edge information. The edge locations at different scales are detected by dividing the image into orientation and scale sub bands obtained by the basis filters.

A two dimensional Gabor filter is defined by

$$
\begin{aligned}
k(x, y) & =h(x, y) \exp (2 \pi j w x) \\
& =\frac{1}{2 \pi \sigma_{x} \sigma_{y}} \exp \left(\frac{-1}{2}\left(\frac{x^{2}}{\sigma_{x}^{2}}+\frac{y^{2}}{\sigma_{y}^{2}}\right)\right)
\end{aligned}
$$

Let $\mathrm{I}(\mathrm{x}, \mathrm{y})$ is an image with size $\mathrm{m} \mathrm{x} \mathrm{n}$. The gabor filtered output $\mathrm{K}(\mathrm{x}, \mathrm{y})$ of the image $\mathrm{I}(\mathrm{x}, \mathrm{y})$ is defined as its convolution with the gabor filter $\mathrm{k}(\mathrm{x}, \mathrm{y})$.

The procedural steps for texture feature extraction are

1. Convert the given image from RGB to Ycbcr color space.

2. Convolve the Gabor filter and Luminance component Y.

3. Gabor filter decomposition is performed by considering three scale and four orientations $\left(0^{\circ}, 45^{\circ}, 90^{\circ}, 135^{\circ}\right)$. The image regions with dominant orientations are identified in this step.

4. Non-Linear operations and smoothing are applied as post processing steps.

5. The maximum value of the four Gabor coefficients at any pixel $(i, j)$, is denoted by the texture feature $\mathrm{TF}_{\mathrm{i}, \mathrm{j}}$.

6. The spatial information is embedded into the feature vector in the form of pixel $\mathrm{x}$ and $\mathrm{y}$ coordinates at the corresponding pixel location. The pixel level spatial feature is defined as

$$
\left.S F_{i j}{ }^{k}=(i / \max (i), j) / \max (j)\right)
$$

\subsection{Justification for using the above Algorithm}

The proposed algorithm uses the complementary aspects and advantages of Soft Sets, Rough Sets, and Fuzzy Sets which all deal with uncertain data such as an image. It has been experimentally found that Soft Rough Fuzzy Set Clustering performs better compared to the other algorithms found in the literature and the above clustering algorithm has not been explored for image processing applications. The training data obtained by clustering using SRFCM is fed to SMO-SVM. The SMO-SVM works by dividing a large task into subtasks, and hence can work efficiently as a classifier on large data sets and is well suited for colour images which contain 
Signal \& Image Processing : An International Journal (SIPIJ) Vol.6, No.5, October 2015

three dimensional data. The linear SVM was experimentally found to not work well for large data sets such as colour images and does not converge, but the SMO SVM is found to converge for large data sets such as digital images, colour images in particular. Hence the SMO SVM with a radial basis function kernel (rbf) was used in this experiment.

\section{Performance Measures}

There exists many segmentation evaluation measures in the literature viz [4] sensitivity, specificity, Precision, Recall, ROC, F-measure, Local consistency Error, Global consistency Error etc. The Performance measures proposed by Unni Krishnan et al., [15] which are Rand Index (RI), Variation of Information (VOI), Global Consistency Error (GCE), and Boundary Displacement Error (BDE) are used in evaluating and comparing our segmentation results with benchmark algorithms.

\subsection{Rand Index}

The Rand index indicates the proportion of pixels which are in agreement between the Computed Segmentation (CS) and the Ground Truth (GT). [15].The rand index is given by the formula

$$
R=\frac{A+B}{A+B+C+D}
$$

$A$ - The pairs of pixels that are in similar sets of CS and GT.

$B$ - The pairs of pixels that are in dissimilar sets of CS and GT.

$C$ - The pairs of pixels that are in similar sets of CS and dissimilar sets of GT.

$D$ - The pairs of pixels that are in dissimilar sets of CS and similar sets of GT.

$A+B$ is the number of coincidences between CS and GT and $C+D$ is the number of noncoincidences between CS and GT. The rand index ranges between 0 and 1 , where 0 confirms that CS and GT do not have common attributes and 1 confirms that CS and GT are indistinguishable.

\subsection{Variation of Information}

The variation of information (VOI) is a measure that specifies the variation between computed segmentation and ground Truth. The difference between average conditional entropy of computed segmentation (CS) and Ground Truth (GT) is used to measure the vagueness in CS which cannot be expressed by GT. Let X and Y be the computed segmentation and Ground Truth which are defined as

$$
\begin{array}{r}
X=\left\{X_{1}, X_{2}, X_{3} \ldots \ldots \ldots . X_{k}\right\} \\
\text { and } Y=\left\{Y_{1}, Y_{2}, Y_{3}, \ldots \ldots \ldots \ldots Y_{l}\right\} \\
n=\Sigma\left|X_{i}\right|=\sum_{j}\left|Y_{j}\right| \\
r_{i j}=\frac{\sum\left|X_{i} \mathrm{I} Y_{j}\right|}{n}
\end{array}
$$

The variation of information between CS and GT is given by

$V I(X, Y)=-\sum_{i, j} r_{i j}\left[\log \left(\frac{r_{i j}}{p_{i}}\right)+\log \left(\frac{r_{i j}}{q_{j}}\right)\right]$ 
Signal \& Image Processing : An International Journal (SIPIJ) Vol.6, No.5, October 2015

The lower is the value of VOI, the better is the segmentation result.

\subsection{Global Consistency Error}

Global consistency error is a measure of the limits to which the computed segmentation can be seen as transformation of Ground Truth towards Computed Segmentation. Similar segmentations match, as both have genesis in the same image, but undergo segmentation at different scales. If one segment is proper subset of the other, then the pixel lies in an area of refinement, and the error should be zero. If there is no subset relationship, then the two regions overlap.

The formula for GCE is as follows

$$
G C E=\frac{1}{n} \min \left\{\sum_{i} E(s 1, s 2, p i), \sum_{i} E(s 2, s 1, p i)\right\}
$$

GCE ranges between 0 and 1 where 0 signifies no error . Lower the value of GCE better is the segmentation result.

\subsection{Boundary Displacement Error}

The Boundary Displacement Error is a measure of the displacement error averaged between boundary pixels in computed segmentation and the nearest boundary pixels in the ground truth. BDE should be low for good segmentation.

\section{RESULTS}

\subsection{PICTORIAL REPRESENTATION}

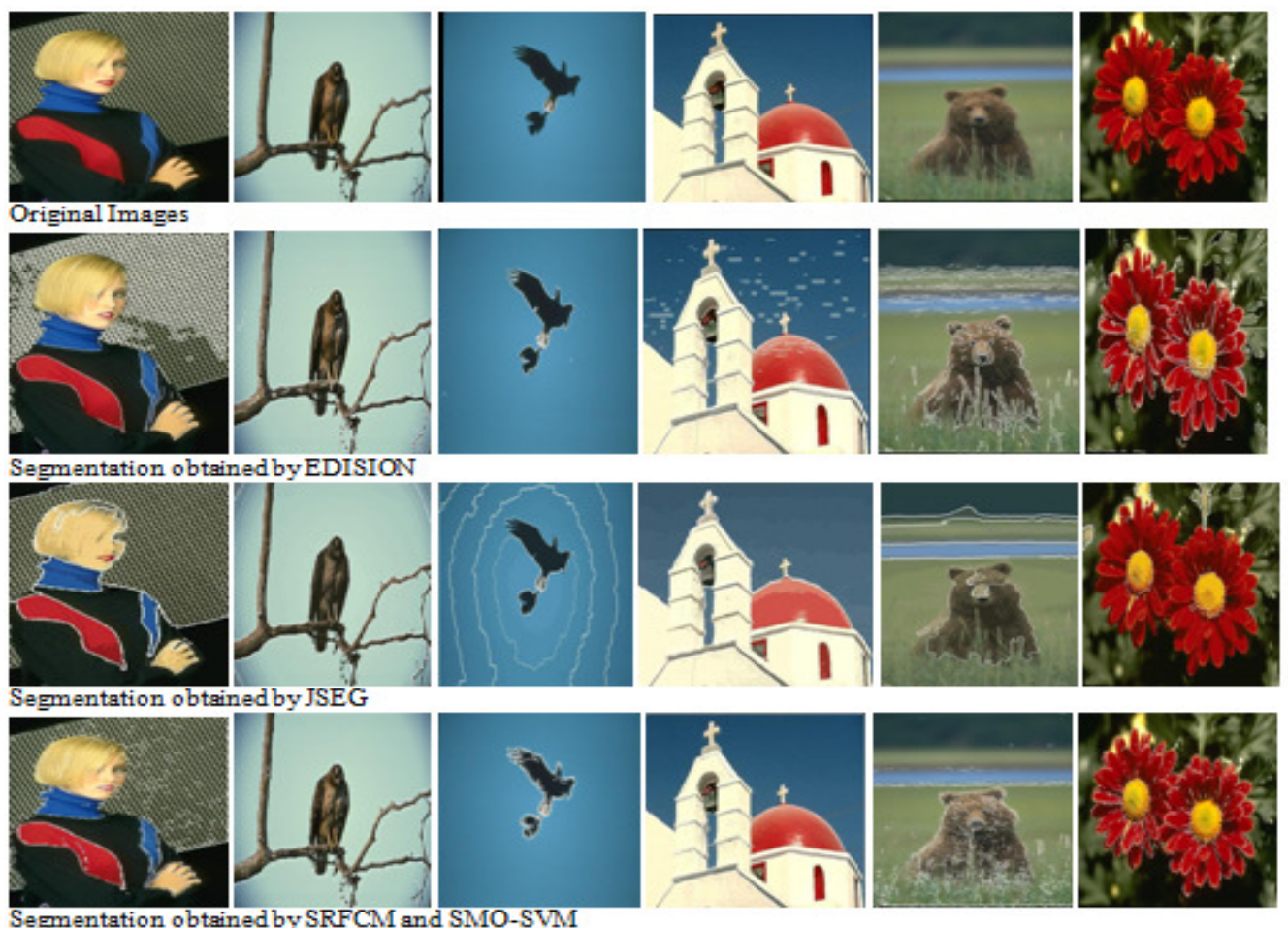


Signal \& Image Processing : An International Journal (SIPIJ) Vol.6, No.5, October 2015
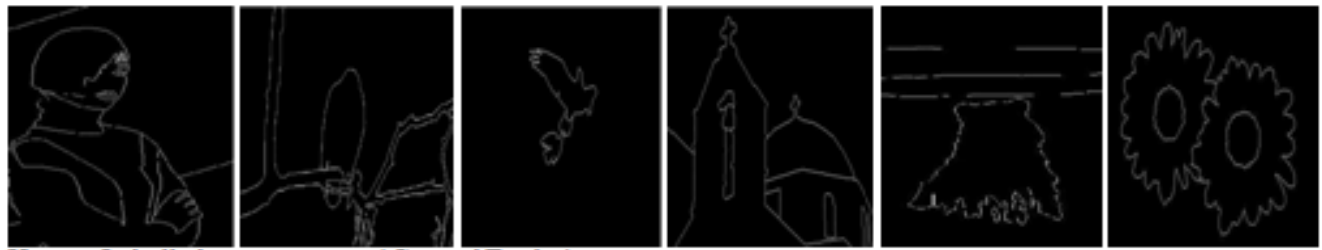

Human Labelled segmentations (Ground Truths)

\subsection{Tabular Representation}

Table 1: Rand Index \& VOI

\begin{tabular}{|c|c|c|c|c|c|c|}
\hline \multirow{2}{*}{ Test Image } & \multicolumn{3}{|c|}{ Rand Index } & \multicolumn{3}{c|}{ VOI } \\
\cline { 2 - 7 } & JSEG & EDISION & SRFCM & JSEG & EDISION & SRFCM \\
\hline Image 1 & 0.5235 & 0.5146 & 0.5350 & 4.8890 & 5.5566 & 4.1155 \\
\hline Image 2 & 0.5783 & 0.4626 & 0.5089 & 5.5364 & 3.5299 & 4.1412 \\
\hline Image3 & 0.5312 & 0.4768 & 0.5328 & 4.6328 & 1.7133 & 1.8906 \\
\hline Image4 & 0.4496 & 0.4804 & 0.4876 & 2.6811 & 3.6909 & 3.7139 \\
\hline Image5 & 0.4630 & 0.5146 & 0.5294 & 2.9197 & 4.1379 & 4.6752 \\
\hline Image6 & 0.4273 & 0.5215 & 0.5369 & 2.7025 & 4.6392 & 4.0036 \\
\hline
\end{tabular}

Table 2: GCE \& BDE

\begin{tabular}{|c|c|c|c|c|c|c|}
\hline \multirow{2}{*}{ Test Image } & \multicolumn{3}{|c|}{ GCE } & \multicolumn{3}{c|}{ BDE } \\
\cline { 2 - 7 } & JSEG & EDISION & SRFCM & JSEG & EDISION & SRFCM \\
\hline Image 1 & 0.5989 & 0.5977 & 0.4492 & 3.5481 & 3.8010 & 4.2550 \\
\hline Image 2 & 0.5962 & 0.2916 & 0.4650 & 1.6188 & 7.8609 & 6.3043 \\
\hline Image 3 & 0.5269 & 0.0596 & 0.1006 & 3.6692 & 29.3927 & 35.0389 \\
\hline Image 4 & 0.1677 & 0.3631 & 0.3862 & 12.8279 & 4.0584 & 3.8659 \\
\hline Image 5 & 0.2561 & 0.4586 & 0.5652 & 7.9991 & 4.6206 & 4.7587 \\
\hline Image 6 & 0.1530 & 0.5398 & 0.4517 & 13.5067 & 2.3773 & 7.8439 \\
\hline
\end{tabular}

The experimental results show the performance comparison of the proposed algorithm, with state of the art JSEG algorithm [19] and EDISION [20] scheme. Six images with colour and texture variance from Berkeley Segmentation database are used for comparison. The algorithms have been implemented in Matlab using P-IV processor system with 4GB RAM. The observations in the table shows that SRFCM out performs the algorithms in [19] and [20] in terms of rand index for images 4,5 and 6. It can be observed that the proposed algorithm exhibits better performance results for most of the images in terms of RI, VOI, GCE and BDE. 
Signal \& Image Processing : An International Journal (SIPIJ) Vol.6, No.5, October 2015

\section{CONCLUSION}

The developed approach is a robust technique which integrated the strengths of three soft computing techniques which are rough sets, soft sets and fuzzy sets and applied the obtained results to the well known machine learning tool, SMO-support vector machine for segmentation. Extensive Experimentation has been done on a lot of images from Berkeley segmentation database which consists of 500 natural color images along with their Ground Truths. The effectiveness of proposed algorithm is demonstrated along with the comparison with other state of the art algorithms. The results shows that in soft rough fuzzy c-means clustering with SMO support vector machine inter cluster distance has been maximized and intra clustering distance has been minimized. Various performance metrics have been compared and the proposed algorithm shows better results compared with other existing benchmark algorithms. The proposed algorithm can also be extended to multi class support vector machine which increases the convergence speed. The proposed algorithm can also be used with noisy color images.

\section{ACKNOWLEDGEMENT}

The first author would like to thank Dr. Ch. Srinivasa Rao, Professor \& HOD, Department of E.C.E, JNTUK, Vizianagaram, Andhra Pradesh , India for his valuable inputs and suggestions, the technical and administrative Staff of Department of ECE, JNTUK, Vizianagaram for providing the lab facilities in the Department.

\section{REFERENCES}

[1] Cheng H.D, Jiang X.H, Sun Y, and Wang. J, "Colour Image Segmentation: Advances and prospects" The Journal of Pattern Recognition Society (2001)2259-2281.

[2] Christoudias.C.M, Georgescu. B., andMeer. P. "Synergism in low-level vision" in 16th I.E.E.E conference, Pattern Recognition,vol.4,pp 150-155,IEEE,New York City,New York

[3] Deng.Y, Manjunath. B.S, "Unsupervised Segmentation of color-Texture regions in Images and Video" IEEE Transactions on Pattern Analysis and Machine Intelligence VOL.23, NO.8, 2001.

[4] Dana E.I.,Paul F.W,'Image Segmentation based on the integration of colour texture descriptors-A review" Elsevier,PatternRecognition,44 (2011) pp2479-2501.

[5] Freixenet. J, Munoz, Marti. J, Llado, Colour texture Segmentation by region boundary cooperation, Proceedings of the European Conference on Computer Vision 2(2004),250-261.

[6] John. C. P, "Sequential Minimal Optimization, A Fast Algorithm for Training Support Vector Machines Microsoft Research. A Technical Report” MSR -TR-98-14 1998.(SMO)

[7] Jain A. K, "Unsupervised texture segmentation using Gabor filters". Farrokhnia, F.,IEEE International conference on systems, Man and cybernetics, 1990

[8] Lingras P, and West C, "Interval set clustering of web users with rough k-means Journal of intelligent information systems".Springer-2004.

[9] Majumdar. P, Samantha S.K , "On similarity measures of fuzzy soft sets" International journal of advanced soft computing applications 2011.

[10] Mitra. S, Banka. H, and Pedrycz, "Rough Fuzzy Collaborative clustering" IEEE Transactions on systems, MAN AND CYBERNETICS-VOL.36 NO.4 2006.

[11] Mushrif. M.M, Sengupta.S, Ray AK "Texture classification using a novel soft set theory based classification algorithm" ACCB Springer 2006.

[12] Mushrif M.M, and Ray A.K,"A-IFS Histon based multithresholding algorithm for colour image segmentation.

[13] Pawalak. Z, "Rough sets, Theoretical aspects of Reasoning about data". Dordrecht, The Netherlands: Kluwer, 1991. 
Signal \& Image Processing : An International Journal (SIPIJ) Vol.6, No.5, October 2015

[14] Rocio A. L-Morales, Raul E. S-Yanez, Victor A-R, and Fernando E. C, ”Integration of color and texture cues in a rough set based segmentation method" SPIE Journal of Electronic Imaging 23(2).

[15] UnniKrishnan, pantofaru. C. and Hebert. M.," Towards objective evaluation of Image Segmentation Algorithms."IEEE Transactions on Pattern Analysis and Machine Intelligence" Vol 29,no 6,pp 929944,June 2007.

[16] Venkataramana Reddy.B.D, T.Jayachandra Prasad, "Colour-Texture segmentation using Hyper Complex Gabor Analysis", SIPIJ,AIRCCSE,Volume 1, Number 2, December 2010.

[17] Wang X, Sun Y,"colour- and texture-based image segmentation Algorithm”, Machine Graphics \& Vision 19 (1)-2010 3-18.

[18] Wang. L, "Support vector machines: theory and applications" Springer, Berlin 2005.

[19] Wang. X.Y, Wang. T, Juan B" Colour Image Segmentation using pixel wise support vector machine", Pattern Recognition 44 (2011) 777-787

[20] Ying.H, Yang, Wang. X.Y, Wang Q.Y, Zhang.X.J "LS-SVM based image segmentation using color and texture information", Journal of Visual Communication and Image Representation, Vol 23, October 2012.

[21] Zadeh L. A, "Fuzzy logic, neural networks, and soft computing commun.CM" vol.37 No.3 pp.77-84 Mar.19

\section{AUTHORS}

RVV Krishna received B.Tech from Nagarjuna University A.P, India in Electronics and Communication Engineering and Master's Degree in Digital Systems and Computer Electronics from JNTU Hyderabad in 2002.He is currently pursuing Ph.D in JNTUK, Kakinada. His area of interest is Digital Image Processing.

E-mail : rvvkrishnaece@gmail.com

S Srinivas Kumar received Ph.D degree from IIT Khargpur. He is presently working as Professor in JNTU College of Engineering, Kakinada and Director R \& D. He has more than 20 years of teaching and research experience. He published 30 papers in international and national Journals. His area of interest is Digital Image Processing and Video Processing.

Dr. Srinivas Kumar is a Fellow of IETE

E-mail : samy_ssk2@yahoo.com
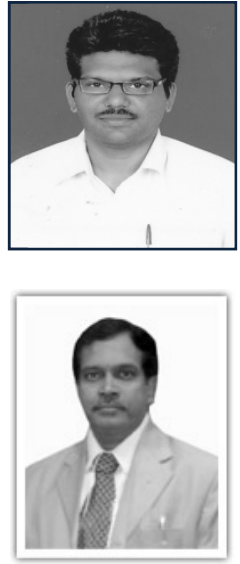